\title{
Controller Design for ISG Hybrid Electric Vehicle Based on SAE J1939 Protocol
}

\author{
Li Yongzhong \\ Dept. of Aeronautical Ammunition Engineer \\ The First Aeronautical College of Air Force \\ Xinyang, China \\ e-mail: lyzsyljw@sina.com
}

\author{
Ji Xinjie \\ Dept. of Aeronautical Ammunition Engineer \\ The First Aeronautical College of Air Force \\ Xinyang, China \\ e-mail: jixnjie@126.com
}

\begin{abstract}
During the design of IVECO ISG hybrid electric vehicles (HEVs), the ISG electrical propelling system was inserted into the Duling IVECO vehicle. Thus the controller of ISG electrical propelling system constructed the distributed control system with the other controller. However, field bus is one of key techniques in the distributed control system. Firstly, CAN communication protocol for ISG controller based on the SAE J1939 CAN bus protocol was presented, then the hardware and software of ISG controller was designed in modular.
\end{abstract}

Keywords-hybrid electric vehicled; controller; integrated starter/generator; CAN bus; SAE J1939 protocol; design

\section{OUTLINE OF SAE J1939 CAN Bus}

CAN bus is high credibility, vivid and real-time the field bus (Field Bus). The biggest characteristics of CAN protocol is to abolish the traditional station address coding, but to replace it with the communication data block coding. This coding format can make the different nodes receive the same data at the same time and get away from the point to point stipulation, so it's vivid to constitute different distributed measure and control system. At the same time, the adoption of the communication data block coding can make the node's number of the network theoretically free from restriction, which is very important for the distributed measures and control system. In CAN protocol, the most length of the data segment is 8 bytes, this can meet the general demands of industrial control order, work status and test data. And 8 bytes won't take up too long bus time, while promising the real-time of data communication.

The CAN protocol adopts CRC check, and it provides false processing function, this can guarantee the credibility of data communication. The structure of CAN bus is simple, it connects with the external world only by two lines. CAN bus is a kind of multi-master bus, all the nodes can send out an information to the other nodes in the network all the moment. In summary, CAN bus is of the most credibility, vivid, real-time, anti-interference ability and simple structure, so it's generally accepted for the most prospective filed bus.

The SAE J1939 protocol is the applied layer protocol based on CAN bus 2.0B, which aims at the truck and big passenger bus. CAN 2.0B protocol includes two kinds of message format, standard frame and expand frame, but the SAE J1939 protocol has to adopt the expand message format. Based on CAN bus 2.0B, the SAE J1939 protocol gives the further definition of 29 arbitration field identifiers in expand frame message. Being compatible with the standard message format, the standard message is allowed to use in the local area network (LAN) of SAE J1939 protocol.

In the SAE J1939 protocol, the Protocol Data Unit (PDU) is used to pack the delivered data. PDU is packed in one or more CAN data frames, and delivered to the other network devices through a physical medium. The format of PDU is shown on TABLE I.

TABLE I.

SAE J1939 PDU FORMAT

\begin{tabular}{|c|c|c|c|}
\hline P & PGN & SA & DF \\
\hline $\boldsymbol{P}$ & $\boldsymbol{R}$ DP $\boldsymbol{P F} \boldsymbol{P S}$ & $\boldsymbol{S A}$ & $\boldsymbol{D F}$ \\
\hline 3 bits & 1 bit 1 bit 8 bits 8 bits & 8 bits & $0-64$ bits \\
\hline
\end{tabular}

II. CAN BUS COMMUNICATION PROTOCOL OF ISG CONTROLLER

CAN communication protocol of ISG (Integrated Starter/Generator) controller is one part of the distributed control system protocol of IVECO hybrid electric vehicle, Fig. 1 is the structure of the system protocol.

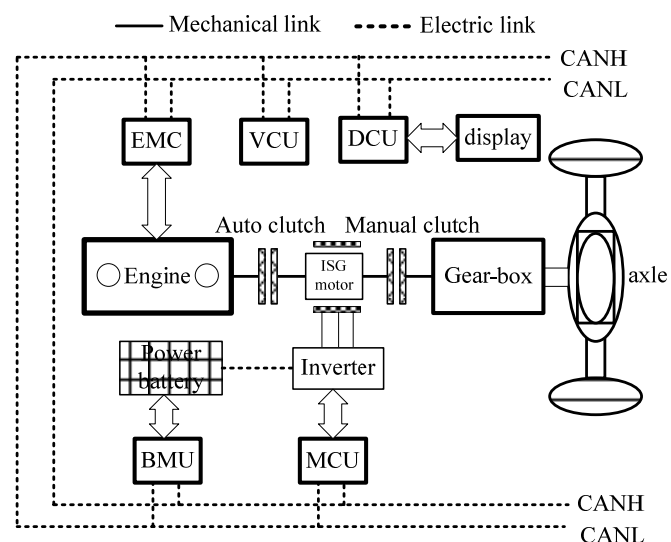

Figure 1. Distributed control system of IVECO hybrid electric vehicle

According to the actual circumstance, the communication protocol of ISG controller is drawn up by the following steps:

a) Group the Messages. When the parameters including in the node message is less, the message can be transmitted by the single data frame. But, if the parameters including in the node message is more, the single message can't meet the demands, so it's needed to classify the 
parameters, and program them into different data frame according to its importance. There're many kinds of message parameters transmitted to the network by ISG controller, they are as follows mainly: rotating speed of ISG motor, estimated torque, most usable torque, power of ISG motor, mechanical power, direct voltage, direct current, temperature of motor and inverter, overcurrent and overvoltage signal etc, it's needed to group and code these parameters, and then transmit them to the network. The messages are grouped into 4 groups.

b) Choose the Transmitting Period of the Message. The transmitting frequency is decided by the importance of the information and the updating degree of the information quantity. The ISG controller broadcasts its states to CAN bus network continuously, such as the ISG rotating speed, torque, voltage, current and work mode etc. According to the importance of the data, the transmitting period of data frame is set to $10 \mathrm{~ms}, 100 \mathrm{~ms}, 1 \mathrm{~s}$ in turn, this can avoid a bus competition.

c) Choose Message Priority. There're 8 priorities in all, and they occupy the front three places, the top is 0 , the lowest is 7. In default state, the priority of all control message is set to 3 , the priority of the other message, such as specified message, request message, answer message, is set to 6 . Because the message broadcasted to network by ISG controller is very important for the VCU control decision, so the priority of the ISG node message is set to 3 .

d) Choose the Network Node Address. In the CAN network, the node address is unique. An address is correspondent to the particular device's node in the network. The network address recommended in the SANE J1939 protocol is chosen as the network node address in this paper, the ISG controller node address is defined as 31.

e) Choose the Message Transmitting Way. The transmitting way of the CAN message includes: point to point transmitting, broadcast transmitting, the period transmitting and request transmitting. The data of ISG controller is transmitted to the VCU node through the network. In consideration of the control function between the VCU and the ISG controller, the transmitting way of point to point is chosen here.

f) Code the Message. After the above steps are completed, the message frame of ISG controller can be coded according to the J1939 frame format.

In the designing process of ISG controller in IVECO hybrid electric vehicles, the ISG controller messages are grouped into 4, their coding difference lies in the PDU code, the communication period and the packed data. The PDU codes of 4 groups of messages are: 0xcffl01F, 0xcffl11F, $0 \mathrm{xcffl} 21 \mathrm{~F}$ and $0 \mathrm{xcffl} 3 \mathrm{~F}$.

\section{The MOdUlarization Structure ANALYSIS OF ISG CONTROLLER}

The function of ISG controller mainly includes: receive and transmit data; compute control instruction; drive ISG motor; overcurrent protection; produce multiple insulating power; acquisition and processing of sensor signal etc. Fig. 2 is the main function modules and signal-flow diagram of ISG controller.

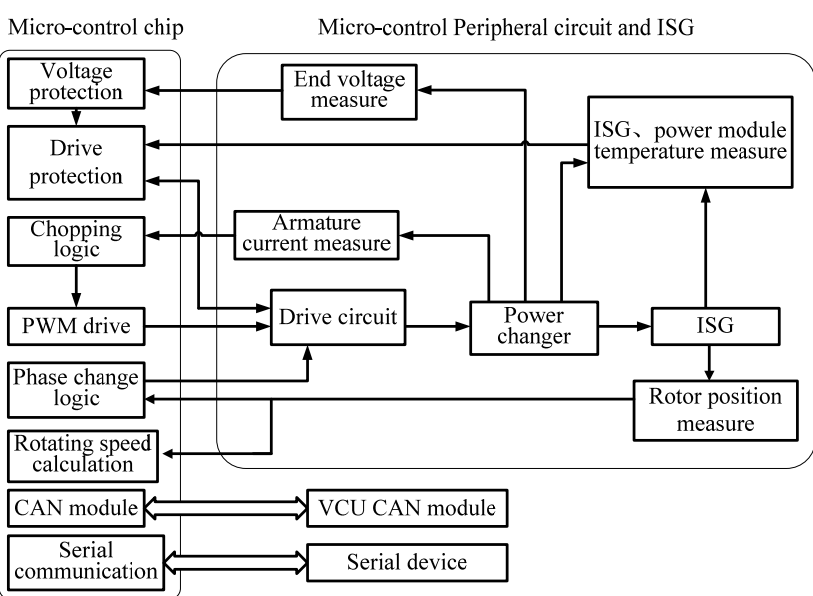

Figure 2. Main function modules of ISG controller

\section{The Modularization Design OF ISG CONTROLleR HARDWARE}

The main modules of ISG controller includes microcontroller module, CAN bus communication module, signal adjusting and A/D sampling module, power module, PWM drive module, rotor position measuring module and multiple insulating power module etc, only two main modules related to CAN bus communication are introduced here for lack of space.

\section{A. Design of the Micro-controller Module}

Digitalization and bus construction of ISG controller is the inevitable trend of vehicle's electric apparatus. In consideration of low cost, credibility, small physical volume, bus communication etc, the DSP technique is used in this paper. There are many kinds of DSP or MCU chips, and their difference is big, but only the motor control chip, developed by TI, AD, MITSUBISHI and Microchip Company, is suitable for the motor situation, and it's of development prospect. The Microchip's dsPIC30F6010A is chosen as the core micro-controller module.

\section{B. Design of CAN Bus Module Interface}

According to the design principle of improving the antiinterference ability, the photoelectric isolation is adopted in the interface circuit of CAN bus, and it's also used to amplify the signal. At the same time, the PCA82C250 transceiver is used between the micro-controller CAN module and physical bus, it can offer the differential transmitting ability to bus, and the receiving ability to CAN controller. 


\section{The Modularization Design of ISG CONTROller SOFTWARE}

The main functions of the ISG controller software include: initialization of variable, initialization of chip's functional module, initialization of $\mathrm{I} / \mathrm{O}$, interruption treatment, PWM drive of motor, current measurement, voltage measurement, chopping control, position catch, calculation of rotating speed, overcurrent protection of power transistor, receive of VCU order, and transmit of ISG state information etc. For the sake of the processing efficiency of events, the software structure of ISG controller adopts coordination work of main program and interruption handling program. The main program is mainly responsible for software's initialization, the motor speed calculation, the status display etc, the interruption handling program is responsible for the rest events. Therefore, the ISG controller software is divided into a main program and 5 interruption handling programs according to the demands, the software structure is shown as Fig. 3.

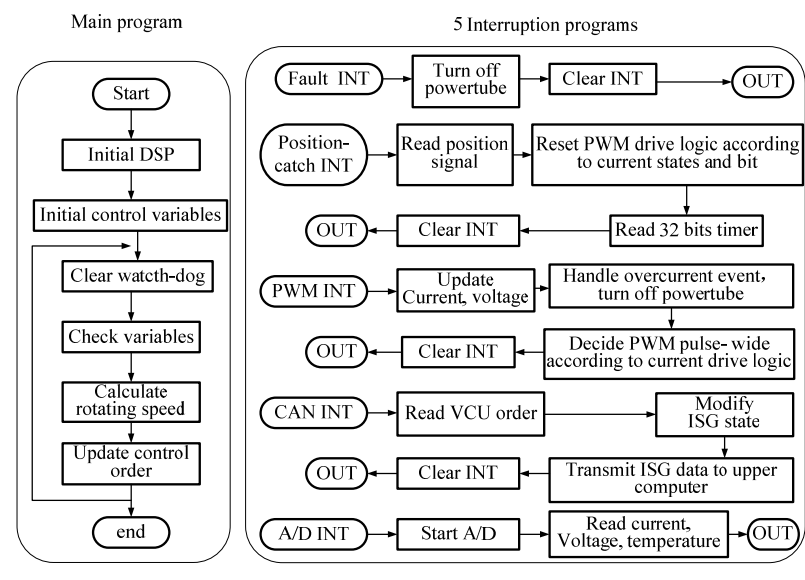

Figure 3. Software structure and flow of ISG controller

\section{The PROtOTYPe MACHINE OF ISG CONTROLLER}

According to the SAE J1939 communication protocol, the hardware and software of ISG controller is designed, the ISG controller has been developed, and the corresponding control circuit boards have been produced. ISG controller and circuit boards are shown as Fig. 4 and Fig. 5.

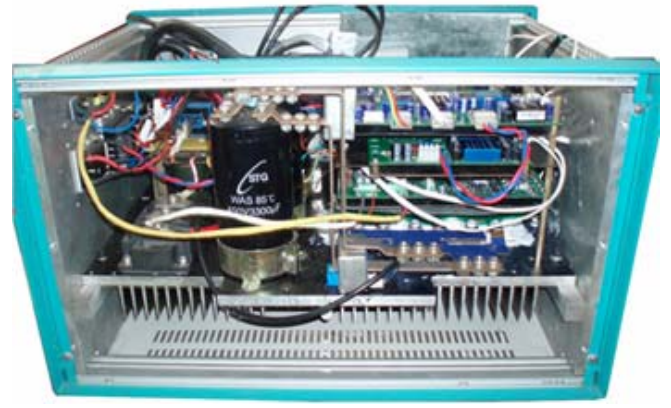

Figure 4. Appearance of ISG controller

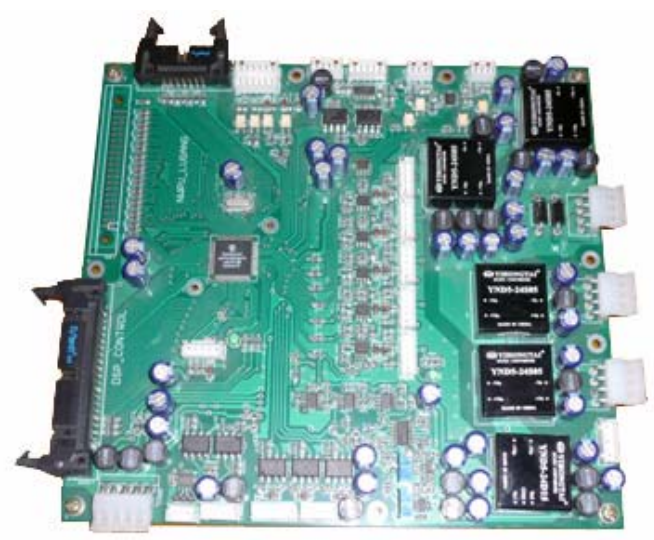

Figure 5. Circuit board of ISG controller

\section{CONCLUSIONS}

By analyzing function modularization of ISG controller in hybrid electric vehicle, the ISG controller meeting SAE J1939 CAN protocol is designed for ISG electric driving system. This controller is of complete function and stable performance in rack testing and whole vehicle debugging.

\section{REFERENCES}

[1] R. V. Chacko, Z. V. Lakaparampil, V. Chandrasekar. "CAN Based Distributed Real time Controller Implementation for Hybrid Electric Vehicle”, Proc. IEEE Conference on Vehicle Power and Propulsion, 2005, pp. 247-251.

[2] A. Sciarretta, L. Guzzella. Control of Hybrid Electric Vehicles [J]. IEEE Control Systems Magazine, 2007, 27(2), pp.60-70.

[3] W. Cai. "Comparision and Review of Electric Machines for Integrated Starter Alternator Applications" [C]. Proceedings of the IEEE IAS Annual Meeting, 2004, pp. 386-393.

[4] BOSCH CAN Specification, Version 2.0, 1991.

[5] SAE J1939-81, Network Management. July 1997.

[6] SAE J1939-21, Data Link Layer, July 1994. 